\title{
2009 College of Biological Sciences Poster Day Abstracts
}

\author{
The following abstracts were prepared by students participating in the \\ 2009 College of Biological Sciences Poster Day at the College of Biological Sciences
}

\section{In silico studies of MBP segments in lipid membranes \\ Kirill Bessonov, George Harauz \\ Molecular and Cellular Biology, College of Biological Sciences}

Our central nervous system is a dynamic and complex system primarily composed of neuronal and glial cells. Oligodendrocytes have long processes that wrap around nerve axons, forming myelin sheath, and allowing for rapid signal propagation. The CNS myelin sheath is composed of many layers that are maintained by myelin basic protein (MBP) and proteolipid protein (PLP). The role of MBP is not only to act as a molecular linker of myelin, as its different isoforms had been found to regulate T-cell activation, $\mathrm{Ca}^{2+}$ influx, signal transduction in myelination and demyelination. The most abundant and conserved MBP form in mammals of $18.5 \mathrm{kDa}$ under physiological conditions, the protein has a net charge of +19 , is intrinsically disordered, and contains a low proportion of hydrophobic residues. MBP also shows a large number of post-translational modifications (PTMs) including deimination, phosphorylation, deamidation, methylation, and $\mathrm{N}$-terminal acylation that influence the overall charge and possibly function. Multiple Sclerosis (MS) is a complex neurodegenerative disease diagnosed in young adults, aged 15 to 40, affecting vision, hearing, memory and movement. In MS, axons are demyelinated and slowly form inflammatory lesions. As the disease progresses, oligodendrocytes and, ultimately, the axons themselves are destroyed. Reduction in the net positive charge of MBP, through deimination and possibly other PTMs, correlates strongly with disease severity and may mediate myelin instability and loss of compaction. The C-terminal A141L154 MBP alpha-helical segment (AYDAQGTLSKIFKL) as determined by hydrophobic moment plot was simulated in silico using Gromacs software in neutral membrane box containing DMPC lipids and water as solvent for $200 \mathrm{~ns}$. Partial uncoiling and recoiling of alpha-helical region was observed, overall, the alpha-helix showed significant stability. The A141-L154 segment extensively interacted with DMPC head groups: a) the Tyr142 and Phe152 residues are highly dynamic, preferring the hydrophilic environment of lipid head groups; b) non-polar Phe152 is completely embedded in the hydrophobic $\mathrm{CH}$ chains; c) positively- charged Lys151 and Lys154 interact with the DMPC head groups, pulling and anchoring the A141-L154 peptide to the membrane.The overall dynamic nature as well as relative role of each residue of the segment was appreciated. In silico simulations were useful to assess relative stability, membrane interactions/penetration depth, and to confirm the amphipathic nature of the A141-L154 segment. In silico simulations provide a novel way of acquiring knowledge on MBP topology and function in lipid membranes, thus extending our understanding of MS pathogenesis.

Comparative analysis of rapid responses of mitochondria and peroxisomes to oxidative stress

Elyse Roach, Jaideep Mathur

Department of Molecular and Cellular Biology, College of Biological Sciences

Plants combat diverse environmental stresses while remaining rooted to a spot. Plant cells clearly possess the molecular and cellular biological machinery required for responding rapidly to stress and minimizing its negative effects. Mitochondria and peroxisomes are major organelles involved in the production and scavenging of reactive oxygen species (ROS). As part of their stress-response, both organelles produce tubular protrusions called matrixules and peroxules, respectively. These transient extensions have been observed separately and it is unclear whether a response hierarchy exists between the two organelles or their responses occur independently. Through a series of experiments with transgenic Arabidopsis thaliana plants, we analyzed the relative responses of mitochondria and peroxisomes to hydrogen peroxide, a ROS that results in the generation of toxic hydroxyl radicals. Exogenous $\mathrm{H}_{2} \mathrm{O}_{2}$ application at $0.3 \%, 3.0 \%$, and $30 \%$ for 45 seconds resulted in $33.4 \pm 9.6,75.2 \pm 3.8$ and $0 \%$ elongated mitochondria and $12.05 \pm 2.95,59.6 \pm 7$, and $77.5 \pm 3.5 \%$ elongated peroxisomes, respectively. Similarly, under high intensity light (200umol $/ \mathrm{m}^{2} / \mathrm{sec}$ ) treatments of $5,10,15$ and 20 minutes 30 $\pm 2,46 \pm 2,80 \pm 4,10 \pm 2 \%$ elongated mitochondria and 0,6 $\pm 2,10 \pm 2$, and $54 \pm 4 \%$ elongated peroxisomes, respectively, were observed. Treatment with varying concentrations of abscisic acid, which also elevates cellular levels of $\mathrm{H}_{2} \mathrm{O}_{2}$, showed similar trends. It was concluded that 
a hierarchy of organelle response exists in which mitochondria begin to produce matrixules at lower levels of oxidative stress and reach a maximum size before dividing by fission. By contrast, peroxisomes do not exhibit peroxule extension until after mitochondria have fully elongated. Under high oxidative stress, peroxisomes elongate to form beaded string-like structures before fragmenting. These studies were crucial in understanding the rapidity of intercellular responses in plants and have paved the way for a subsequent molecular dissection of the phenomenon.

\section{Characterization of potential small molecule inhibitors against mono-ADP ribosyltransferase toxins \\ Sarah Legree, A.R. Merrill \\ Molecular and Cellular Biology, College of Biological Sciences}

Inhibitors of bacterial toxins are ideal alternatives to antibiotics due to their ability to reduce the virulence of bacterial pathogens while also preventing the development of resistance. Mono ADP-ribosyltrasferase toxins (mARTs) are enzymes which catalyze the cleavage of nicotinamide from $\mathrm{NAD}^{+}$and transfer ADP-ribose to a protein target. The purpose of this study was to conduct compound screening followed by characterization of small molecule inhibitors of two mART toxins, $P$. aeruginosa ExoA and $V$. cholerae cholix toxin. Initially, a virtual screen was performed on a library of one-million compounds for inhibitors of cholix toxin, resulting in 31 potential inhibitors. A cell-based screen in yeast was then conducted to identify the best lead compounds, followed by a mammalian cell-based assay. Inhibitor 23 was found to be the most effective against ExoA in yeast, however inhibitors 30 and 12 also protected the mammalian cells from ExoA-induced cell death. Inhibitor $\mathrm{K}_{\mathrm{d}}, \mathrm{T}_{\mathrm{m}}$ (a measure of cholix stability), and $\mathrm{IC}_{50}$ values were then determined for the most active compounds. Although compounds 12 and 23 showed inhibitory activity, compound 30 was the most promising due to its ability to protect mammalian cells from high ExoA doses. Inhibitor 30 stabilized cholix folded structure by $2.9{ }^{\circ} \mathrm{C}\left(\Delta \mathrm{T}_{\mathrm{m}}\right)$ and gave an $\mathrm{IC}_{50}$ value of $3249 \pm 1 \mathrm{nM}$. In summary, inhibitor 30 is a promising lead compound for further optimization in the quest to develop novel therapeutics against mART toxins, which are commonly involved in opportunistic bacterial infections.

\section{Phosphorylation of cardiac regulatory proteins in rainbow trout}

Andrew Robertson, T.E. Gillis

Integrative Biology, College of Biological Sciences

Cardiac contraction is regulated by the cardiac troponin (cTn) complex, composed of cTnC, cTnI, and cTnT. This acts as the $\mathrm{Ca}^{2+}$ activated trigger of cardiac contraction.
Phosphorylation of the subunits affects various responses in the heart, however, cTn phosphorylation can be maladaptive, as it is indicated in myofilament dysfunction seen in heart failures. Rainbow trout was used as a model to understand the effects of cTn phosphorylation on the diseased heart. The trout heart is unique in that it is very sensitive to $\mathrm{Ca}^{2+}$ and has a form of cTnI that is missing two critical phosphorylation sites seen in mammals. In this study, we explored whether trout cTnT (ScTnT) and ScTnI are phosphorylatable and, if so, how many phosphorylation sites they contain. Western blotting, SDS-PAGE, Pro-Q phosphoprotein staining, and densitometry analysis were conducted to observe if ScTnT and ScTnI are phosphorylated. Myofilament protein was also run on 2D-PAGE and western blotted to observe the number of phosphorylation sites on ScTnI and ScTnT. The results from our western blotting revealed that there are actually two isoforms of ScTnT being expressed in the trout heart. Furthermore, densitometric analysis revealed both isoforms have an observed phosphorylation level that is significantly equivalent to phosphorylated standards. The observed phosphorylation level of ScTnI was statistically comparable to nonphosphorylated standards. However, 2D-PAGE analysis has revealed at least two separate spots for ScTnI, suggesting at least one phosphorylation site exists. Further work needs to be condicted to locate ScTnT on 2D-PAGE and elucidate what the observed ScTnI spots represent.

\section{Ultrastructural and live imaging aided dissection of cell-cell dissociation in ARP2/3 complex mutants of Arabidopsis thaliana \\ Natalie Dunn, Ana Donoso, John Greenwood, Jaideep Mathur \\ Molecular and Cellular Biology, College of Biological Sciences}

The seven-subunit Actin Related Protein (ARP) 2/3 complex is a highly conserved modulator of the actin cytoskeleton in eukaryotes. In plants, mutations in different subunits of the complex result in an aberrant actin organization, the distortion of aerial epidermal trichomes and cell-cell detachment in elongating or laterally expanding tissue. We hypothesize that improper organization of F-actin affects numerous subcellular processes including the balance between exocytosis and endocytosis. We used a combination of electron microscopy and confocal microscopy to carry out a spatial and temporal analysis of cell detachment in mutants in ArpC-4 and ArpC-5 (crooked) subunits and in klunker, a mutant displaying a similar phenotype. Ultrastructural images of klunker and crooked mutants demonstrated complete detachment of previously attached epidermal cells. The gaps between these disconnected cells were found to contain fine filamentous strands. Live imaging of autofluorescent deposits at the curling tips of detached mutant cells and strands suggests that they are composed of callose a polymer of $\beta-1,3$ linked glucans. Callose is know to be associated with cell protection, and its excessive deposition 
may cause loss of connectivity between cells. Ultimately, cells that have lost connection with their neighboring cell loose a sense of polarity and result in uncontrolled growth and callus formation. This work thus identifies the role of actin mediation in maintaining the balance between exocytosis and endocytosis balance and thus determining for the degree of cell-cell adhesion in plants.

\section{Comparative analysis of rapid responses of mitochondria and peroxisomes to oxidative stress} Elyse Roach, Jaideep Mathur

Department of Molecular and Cellular Biology, College of Biological Sciences

Plants combat diverse environmental stresses while remaining rooted to a spot. Plant cells clearly possess the molecular and cellular biological machinery required for responding rapidly to stress and minimizing its negative effects. Mitochondria and peroxisomes are major organelles involved in the production and scavenging of reactive oxygen species (ROS). As part of their stress-response, both organelles produce tubular protrusions called matrixules and peroxules, respectively. These transient extensions have been observed separately and it is unclear whether a response hierarchy exists between the two organelles or their responses occur independently. Through a series of experiments with transgenic Arabidopsis thaliana plants, we analyzed the relative responses of mitochondria and peroxisomes to hydrogen peroxide, a ROS that results in the generation of toxic hydroxyl radicals. Exogenous $\mathrm{H}_{2} \mathrm{O}_{2}$ application at $0.3 \%, 3.0 \%$, and $30 \%$ for 45 seconds resulted in $33.4 \pm 9.6,75.2 \pm 3.8$ and $0 \%$ elongated mitochondria and $12.05 \pm 2.95,59.6 \pm 7$, and $77.5 \pm 3.5 \%$ elongated peroxisomes, respectively. Similarly, under high intensity light (200umol $/ \mathrm{m}^{2} / \mathrm{sec}$ ) treatments of 5, 10, 15 and 20 minutes 30 $\pm 2,46 \pm 2,80 \pm 4,10 \pm 2 \%$ elongated mitochondria and 0,6 $\pm 2,10 \pm 2$, and $54 \pm 4 \%$ elongated peroxisomes, respectively, were observed. Treatment with varying concentrations of abscisic acid, which also elevates cellular levels of $\mathrm{H}_{2} \mathrm{O}_{2}$, showed similar trends. It was concluded that a hierarchy of organelle response exists in which mitochondria begin to produce matrixules at lower levels of oxidative stress and reach a maximum size before dividing by fission. By contrast, peroxisomes do not exhibit peroxule extension until after mitochondria have fully elongated. Under high oxidative stress, peroxisomes elongate to form beaded string-like structures before fragmenting. These studies were crucial in understanding the rapidity of intercellular responses in plants and have paved the way for a subsequent molecular dissection of the phenomenon.

\section{Age-related differences in dual task situations during walking}

Daniel Rappaport, Lori Ann Vallis

Human Health \& Nutritional Sciences, College of Biological Sciences

The likelihood of a fall resulting in injury increases as an individual ages. Typically, falls resulting in injury occur when an individual is simultaneously completing two tasks. This study aimed to reveal some biomechanical differences contributing to this increase in falling risk in older adults. Obstacle avoidance strategies of two populations of subjects (young adults, 18-23 years and older adults, 66-85 years) were analyzed under single (obstacle avoidance) and dual task conditions (obstacle avoidance and recall of a series of numbers). Preliminary analyses revealed age-related decreases in trail toe-to-obstacle distance and trail toe obstacle clearance, as well as increased lead heel obstacle clearance $(\mathrm{p}<0.05)$. Older adults also cleared the obstacle with their trailing toe with a smaller safety margin when compared to younger adults $(p=0.046)$. In related data analyses, angular motion of the trunk and head were investigated. Post hoc analysis revealed that older adults produced more lateral angular motion of their trunk and head (about roll axis) in a dual task situation whereas young adults produced less movement in this plane compared to their respective control trials. Further analyses are required to make definite conclusions, however preliminary findings indicate that older adults use riskier strategies to step over obstacles in the travel path during dual task situations. Future research may include investigating differences in planning strategies for obstacle avoidance during dual task situations as well as examination of the relationship between trunk and head roll and step width to control movement of the body center of mass.

\section{Dual tasking during obstacle avoidance in young and older adults: effects of a spatial memory task}

Lydia Frost, Daniel Rapoport, Lori Ann Vallis

Department of Human Health and Nutritional Sciences, College of Biological Sciences

Falls in older adults are a serious health concern; many of these falls occur by tripping over obstacles or while dual tasking. In this study, young adults (YA: $\mathrm{N}=9$; aged $21.1 \pm$ 1.7 years) and older adults (OA: $\mathrm{N}=8$; aged $75.8 \pm 6.0$ years) completed a series of walking trials, including stepping over an obstacle, and walking over the obstacle while performing a spatial memory (SM) task (directed mental navigation of a point about a grid). Optotrak tracked infared light emitted from markers (IREDs) secured to 
participants' body segments. Head, trunk, and feet motion was tracked by digitizing anatomical landmarks with respect to these IREDs. Data analyses included obstacle clearance gait parameters (e.g. landing distance) and range of head and trunk pitch and roll angular motion. It was found that OA reduced their lead heel and trail toe clearance and landed closer to the obstacle as compared to YA; this may increase their risk of stepping down on the obstacle. OA subjects also had smaller pitch and roll ranges than YA, demonstrating a stiffer control of their body segments. The addition of the SM task caused all subjects to land closer to the obstacle and increase their lead heel clearance, indicating a more cautious clearance strategy, and caused OA to decrease and YA to increase the range of segmental pitch. In general, OA adopted a more cautious strategy by having a tighter control over their head and trunk segments, however their clearance of the obstacle was more risky, especially while dual tasking.

\section{The role of visual stimuli in nest building behaviour of the domestic hen}

Debby Gregory, Victoria Freitas, Grégoy Bédécarrats

Animal and Poultry Science, Ontario Agricultural College

Nest building behaviour in the domestic hen, Gallus gallus domesticus, is considered a behavioural need as it is triggered by internal factors, regardless of the environment. These internal factors include the ovulation of the dominant follicle with the concomitant release of progesterone and estrogen. As a result, frustration can be demonstrated by "stereotypic pacing" when hens are not presented with the opportunity to nest in a secluded place, such as a nest box. In layer cages, hens do not have access to a secluded space but still perform "vacuum nest building" which may result in frustration. To further understand which factors (internal and external) contribute to the expression of nesting, we investigated whether visual stimuli are necessary for the initiation of this behaviour using a strain of genetically blind White Leghorn chickens, known as Smokey Joes. We also examined whether it can be learned from sighted birds through social interaction to compensate for the lack of visual stimuli. Using hens which had been raised in floor pens in groups of 4 , we monitored the behaviour of each chicken allocated to pens of all blind, all sighted, or mixed birds in a randomized complete block fashion. As well, each chicken was given a capsule containing one of four colours of fat soluble dye, which coloured the yolks of the eggs and allowed for determination of individual egg laying locations. Results suggest that while blind birds demonstrate some of the behavioural repertoire, visual stimuli is essential for nest building. Birds in all blind pens demonstrated approximately 7 times more restlessness and feet rotations than birds in all sighted pens by week 4 , however, less than half of the nest box visits. Though blind birds were able to locate nest boxes easily through touch and memory, they laid their eggs randomly on the floor without building a nest $97.7 \%$ of the time, whereas sighted birds made use of the nest boxes and substrates provided $91.7 \%$ of the time. In addition, social interactions with sighted birds were unable to compensate for the lack of sight. Overall, visual stimuli appeared to be necessary to fulfil the behavioural need of nest building and seclusion. However, the lack of sight did not prevent prelaying behaviours, suggesting this phase of nesting is triggered by internal factors. Alternatively, the fact that blind birds did not feel the need to build a nest or lay in nest-boxes may indicate they were already experiencing seclusion. Furthermore, our results support a current state of decreased welfare in caged layer hens, due to the lack of opportunities for seclusion. 